\title{
Total quality management within a health district
}

Howard Nattrass

Winchester District Health Authority has been pursuing a continuous quality improvement or total quality management (TQM) approach for just over two years as one of the national TQM pilot sites. It is being applied to the delivery of services within its provider units as well as to the purchaser-provider relationship. This paper summarises the key features of TQM, illustrates its application to health care, and describes its potential value.

Main dimensions of TQM

There are many versions of TQM, and it is important that an organisation develops and internalises its own approach, drawing upon the principles underlying the various schools of thought. For health care there are three main dimensions:

- The supremacy of "the customer," including the "internal customer"

- Meeting agreed measurable requirements

- Process analysis and improvement.

Running through all these are certain fundamental assumptions about empowerment of people and effective leadership.

\section{CUSTOMER FOCUS}

The term customer jars with many people in the health service. This is understandable, and, when possible, terms such as patient or client are preferable. The difficulty, however, is finding a term which refers to all potential recipients of health care services in their broadest sense - including those who are well and the wider community. The term of customer also helps to describe relationships between people working within an organisation - for example, a ward receiving a supplies service or a general practitioner receiving a hospital based pathology service. These are "internal customers."

The real importance of the concept of the customer, however, is that it underlines the service nature of the relationship and can help to reinforce the ideas of the supremacy of the recipient. TQM emphasises this supremacy everything must be geared towards the customer at all times, and all must flow from this. The health service has a well deserved record of staff commitment to care of patients, but really ensuring that in everything we do, all the time the service is geared around patients' and families' wishes would mark a major step forward.

Accessing customers', or users', or community views has to be the starting point in determining service requirements. The range of opportunities and techniques for doing this within the health service is growing. As well as the more usual approaches such as surveys and focus groups, the district has invested over the past two years in community development work designed to empower and access the views of local people. In this way, for example, the requirements of mental health service users and of carers generally have been identified. Our community participant work is also being done in conjunction with other statutory and voluntary agencies.

A frequent difficulty is reconciling or balancing the views of users and professionals in determining health service requirements. The idea of customer supremacy is not intended to supplant professional opinion and influence. That would be absurd and not in the best interests of the consumer. The extent to which people can really influence the care they receive will obviously vary from case to case. For example, the extent to which someone could influence the care he or she receives while unconscious after a road traffic accident is quite different from that for a carer of an elderly dementing person. Yet we should not make rigid assumptions about what is likely to be possible and desirable in this respect in the future. The situation is changing rapidly, and experience in the United States has shown that patients are able and determined to exercise more influence over aspects of their care than was ever thought possible.

The thrust of health care organisations must be to make ever increasing moves along the spectrum of involvement and empowerment. This may be regarded as either a good thing in itself or as essential if people are to take more control of their own health and wellbeing, thereby helping to reduce the level of dependency on the health service and shifting the emphasis from cure to prevention. How this is achieved in practice and within professional and patient/client relationships is a complex issue. Providing the customer with much more information is certainly important, but other approaches are needed as well. The shift will not occur unless any resistances to the involvement of the user on the part of the service provider are reduced. Service providers need to believe that this is a desirable end.

Fulfilling the first dimension of TQM - the supremacy of the customer - is therefore not as straightforward as it might seem; in practice
Howard Nattrass, district general manager 
it is more difficult in health care than in most commercial organisations, where the idea originated.

MEETING AGREED MEASUREABLE

REQUIREMENTS

The second dimension of TQM is about rigour, clarity, purpose, and continuous improvement. Once the needs of the customer (external and internal) have been identified, decisions have to be made about what is actually going to be provided. It is highly unlikely that all the needs and wishes of the customer can be met within practical constraints, so that negotiation and decisions of priority will be required. These might entail further consultations with customers in some way, but in th and the decision will rest with service providers and authorised bodies such as health authorities.

These agreed requirements have to be precise, clear, and monitorable (which usually means measurable). Vague statements such as "highest quality" or "excellence" serve no useful purpose. The requirements must also be achievable, if challenging. This brings us to the all important idea of continuous improvement - that is, the constant quest and process of making things better, month on month, year on year.

It is in this context that the idea of "standards" does not sit comfortably. The word standard is used in many different ways and can carry the same meaning as measurable requirements. If, however, it means that level of performance which is considered acceptable and applies for some time, then it is likely to be about minimum acceptable standards, and as such is not enough to bring about the continuous and dynamic improvements which are the aim of TQM

PROCESS ANALYSIS AND IMPROVEMENT

The last dimension of TQM is perhaps the most unfamiliar to health service workers, except those who are scientifically inclined.

The essence of process analysis and improvement derives from systems thinking and assumes that we are continually operating within systems - macrosystems, microsystems, and systems within them. Making improvements in services and processes, etc, are dependent on an understanding and detailed analysis of the various systems. Although we usually blame individuals for failure, invariably the fault is in the system. Take, for example, the requirement to reduce orthopaedic inpatient waiting lists. The waiting list position is determined by a number of complex and interrelating systems; clinical management, bed management, the operating theatre, and diagnostic service systems are some of the most important. The processes involved need to be analysed in detail and problems and blockages overcome. Well established tools and techniques for doing this are available for teams of service providers to use. It is a team process because of the range and number of people involved in the interlocking systems.

\section{Putting the dimensions together}

It might seem that there is nothing particularly novel in any of these dimensions, and to an extent that is true. What is different is bringing them together and applying them continuously. Really adopting a TQM approach means doing all these things to an extent we have never done before, and doing them across the whole organisation. To use the jargon, it requires a massive culture shift. The figure shows the main principles of TQM and their interrelations.

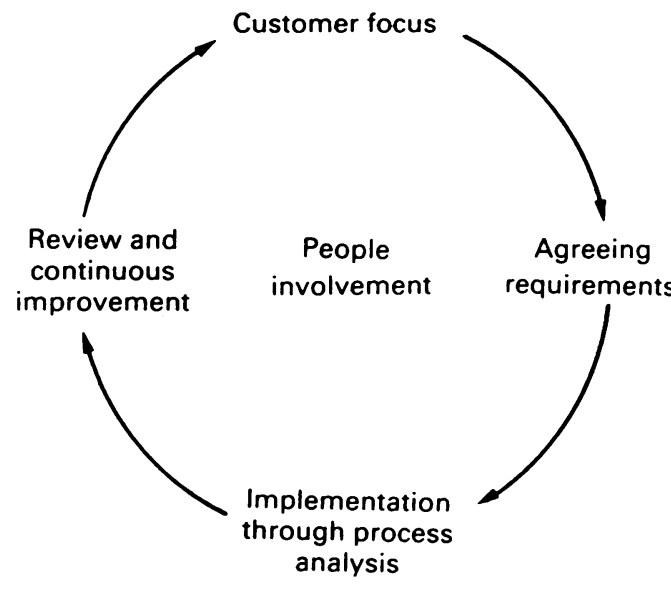

Main principles of TQM

The continuous or total quality approach can provide a coherent framework for "quality" activities in the NHS. In most hospitals or community service units in the United Kingdom the approach to quality lacks any kind of coherence. Medical audit, professional standard setting, external accreditation, consumer satisfaction surveys, contracting for quality, and so forth, are all going on without any integration. I do not mean to devalue this work, which is achieving much of importance in many places. I do believe, however, that by not having a common approach which relates all these activities closely together, we are unlikely to fulfil our enormous potential.

\section{TQM and the organisation}

The TQM approach can apply to any part of the organisation, as well as to the organisation as a whole. It makes as much sense for the clinical team as it does for the purchaserprovider relationship. Within a hospital TQM can apply, for example, to the level of the clinical team or a directorate at the whole hospital. The clinical team is concerned to meet the patient's individual requirements, including those measurable requirements derived from clinical experience and research, such as currently feature in criterion based audit. Protocols, drawn up in conjunction with patients, are a good way of defining requirements. The clinical team is also concerned to meet the requirements of other clinicians - the geriatric department and the orthopaedic department, for example, need to be clear about each other's expectations. None of these elements is static; performance needs to be continuously improved in the light of experience. 
The challenge at this level is in being explicit about what we are aiming at, to ensure it is always customer focused, and to build in the internal customer aspect. The processes people have to analyse and modify are many and complex, and we have often found it difficult to know where to start. Equally, at the level of clinical directorate working through the process of agreeing for whom one is providing a service, to deliver exactly what, and trying year on year to improve can make sense of the whole working of the directorate.

The first thing this highlights in practice is the need to develop leadership and good internal working. None of the aims of TQM can happen unless people are working together and across disciplines. Indeed this is one of the potential benefits of a TQM approach.

\section{TQM and the purchaser-provider relationship}

As a purchasing health authority we are applying a total quality approach in our relationship with our providers in two senses. Firstly, in supporting them to follow a continuous improvement path and, secondly, in viewing our whole relationship as a customer-provider relationship in total quality terms.

In the first sense we are helping our main providers through consultancy support and by paying for training, for example. We are working jointly to identify customer requirements and building into our contracts mainly those areas where we both agree we are seeking further improvement over the coming year. In this way we are aiming at avoiding the type of comprehensive and detailed specifications that are becoming common, which apart from their inherent logistical difficulties seem destined to achieve little more than maintaining the status quo.

To see the whole relationship with providers in a total quality sense is to view it as a longstanding partnership in which both parties support each other to ensure continued improvement. The contrast between this approach and that which many purchasers are taking is well summed up by Don Berwick:

As an incentive to improve quality, the threat of taking one's business elsewhere is pale compared with the reminder that one is committed to a long term relationship. Quality improves as those served (the customers) and those serving (the suppliers) take the time to listen to each other and to work out their inevitable misunderstandings. Just as marriages do not improve under the threat of divorce, neither, in general, will health care. ${ }^{1}$

\section{Other experiences}

Four main conclusions can be drawn from our experience with TQM so far.

Firstly, trying to implememt TQM has been and continues to be hard. We are dealing with a shift in culture, and the prevailing independent professional standards led approach is a strong one. There are so many competing claims on limited time and energy that if TQM is to succeed it will require determined champions, particularly among those in formal leadership positions. One of our mistakes has been not to continue working with the senior management teams until a thorough understanding and commitment was obtained. As a result progress has been patchy.

Secondly, our experience has highlighted a major need to develop leadership at all levels in the organisation, particularly in team working. How people in organisations behave and interrelate is probably the most significant determinant of quality. This is so in most organisations but is especially so in health care, where the nature of the service is so personal. It is my belief that people will treat patients and clients well only if they themselves are well treated and feel valued. Continuous quality improvement or TQM starts from the assumption that people are trying hard already and are acting in good faith. Only if we succeed in involving them will they be able to see to it that everything around them goes well and will their potential and creativity be released. This entails treating each individual with complete respect and, above all, praising, encouraging, and supporting them. It also requires people in formal leadership positions to listen more and to stop feeling they have to know all the answers. We have spent a lot of time developing this new leadership approach, with considerable success.

Thirdly, our experience has confirmed that it is important for people in formal leadership postions to lead in their own behaviour and approach at all times. For example, I now conduct meetings differently, always starting from the viewpoint of what is going well, and in all my daily transactions I try to use language which reinforces the total quality approach.

Finally, a clear lesson for me has been that when people have pursued the total quality approach, whether at the level of a department, clinical team, or small hospital for elderly people, they have greatly enjoyed it and it has made sense to them. It has helped them to make progress.

In the United States many health care organisations are following a total quality approach. I suspect that there, as here, there are major gaps between rhetoric and reality. I would be surprised and disappointed, however, if by the end of this decade most health care organisations in the United Kingdom were not making similar claims.

1 Berwick DM. Continuous improvement as an ideal in health care. $N$ Engl I Med 1989;320:53-6. 